\title{
Europäische lieux de mémoire oder lieux de mémoire für Europa im antiken Griechenland?"
}

\author{
Von \\ Peter Funke
}

Vorderhand erschien eigentlich alles ganz einfach und einleuchtend und in gewisser Weise auch selbstverständlich. Wer mit Blick auf das Ziel einer Konferenz über „,Europäische“ lieux de mémoire?" danach fragte, „ob es so etwas gebe wie ,europäische' lieux de mémoire, also historische Ereignisse oder Prozesse bzw. Symbole oder Persönlichkeiten, die das Zusammenwachsen des Kontinents befördern könnten "“l, der musste doch wohl ganz zwangsläufig - um nicht zu sagen natürlich - auch die Alte Geschichte in seine Überlegungen mit einbeziehen; und auch der befragte Althistoriker sah keine Veranlassung, sich dieser Frage zu entziehen.

Es ist sogar im Gegenteil zu konstatieren, dass sich gerade die Althistoriker in den vergangenen Jahren verstärkt den Fragen einer adäquaten Darstellung einer europäischen Geschichte gestellt haben, die ,mehr sein will und soll als eine Addition von Nationalgeschichten ${ }^{\text {c2 }}$. So wurde 1992 - bezeichnenderweise in Delphi - vom Braunschweiger Georg-Eckert-Institut für internationale Schulbuchforschung eine europäische Konferenz durchgeführt, die nach der Stellung der Antike im Geschichtsunterricht der europäischen Länder fragte und die antike Geschichte nach Paradigmen durchmusterte, die geeignet sein könnten, als Elemente eines Grundkanons für ein in allen europäischen Ländern akzeptiertes Geschichtsbuch für den Schulunterricht zu dienen $^{3}$. Zum gleichen Zweck hat sich 1995 auf Initiative des deutschen Ge-

* Die mir zugedachte Aufgabe, zu Beginn der internationalen Konferenz „Europäische“ lieux de mémoire?" nach solchen „europäischen“" Erinnerungsorten in der griechischen Antike zu fahnden, habe ich genutzt, um durch einen bewusst skeptischen Einstieg in die Diskussion über die Erörterung antiker Spezifika hinaus auch auf die Grundproblematik einer "Europăisierung“ des von Pierre Nora entwickelten Ansatzes hinzuweisen. Der Vortrag wurde daher als ein Impulsreferat konzipiert, dessen essayistischer Stil hier beibehalten wird. Aus diesem Grund und auch angesichts der kaum noch überschaubaren Fülle einschlăgiger Publikationen bleiben die Literaturverweise auf einige wenige weiterführende Titel beschränkt.

1 Zitiert nach einem von Heinz Duchhardt verfassten Thesenpapier, mit welchem der Konferenzrahmen abgesteckt und die der Tagung zugrunde gelegten Arbeitshypothesen skizzenhaft dargestellt werden sollten.

${ }^{2}$ Heinz DUCHHARDT, Thesenpapier (Anm. 1)

${ }^{3}$ Die Ergebnisse dieser Tagung wurden vorgelegt in dem Sammelband: Unity and Units of Antiquity, hrsg. von Kostas Buraselis, Athen 1994. 
schichtslehrerverbandes und einiger althistorischer Fachkollegen die Initiative „Alte Geschichte für Europa“ (AGE) gebildet. Von lieux de mémoire war bei allen diesen Unternehmungen noch nicht die Rede; aber die dort gestellten Fragen zielten zweifellos in die gleiche Richtung, in die wir uns erklärter Maßen auch hier bewegen wollen.

Die Reihe vergleichbarer Aktivitäten ließe sich durchaus noch weiter fortsetzen. Sie sind aber keineswegs in erster Linie einer puren Europabegeisterung entsprungen, sondern haben auch eine sehr pragmatische Seite: Es ist der Versuch, einer in allen europäischen Ländern zu beobachtenden zunehmenden Konzentration des historischen Unterrichtsstoffes auf die Geschichte der neueren und neuesten Zeit und einem damit verbundenen grundlegenden Wandel des Geschichtsverständnisses entgegenzuwirken. Die ideologiekritischen und rezeptionsgeschichtlichen Aspekte dieser Entdeckung und Instrumentalisierung Europas für einen als neu ausgegebenen Geschichtsunterricht böten schon für sich genommenen eine geeignete Ausgangsbasis für die Analyse der Grundlagen einer - oft auch nur vermeintlichen - europäischen Gedächtnislandschaft und ihrer auch von Pierre Nora letztlich geforderten Dekonstruktion ${ }^{4}$.

Ich werde aber diesen Weg nicht beschreiten, der auf eine kritische Durchmusterung des von vielen Seiten immer wieder aufs Neue bereitgestellten Arsenals europabezogener Eckdaten und Schlüsselereignisse aus der Antike hinauslaufen würde. Im abschließenden Teil meiner Ausführungen werde ich zwar auch meinerseits den Versuch unternehmen, zumindest in Umrissen mögliche europäische lieux de mémoire im Bereich der griechischen Antike ausfindig zu machen. Vorab möchte ich aber doch einige allgemeinere Erwägungen in den Vordergrund stellen, um auch das Unbehagen deutlich zu machen, das sich bei mir sowohl auf der Suche nach dem Anteil des antiken Griechenland an einer ,europäischen Geschichte“" wie insbesondere auch im Umgang mit dem dabei zugrunde gelegten theoretischen Ansatz Pierre Noras ${ }^{5}$ eingestellt hat.

Überaus bemerkenswert erscheint mir zunächst einmal die große Selbstverständlichkeit, mit welcher bis heute offenbar allenthalben vorausgesetzt wird, dass die griechisch-römische Antike ein unabdingbarer Bestandteil einer für das heutige Europa konstitutiven Geschichte ist. Die Reihung der Zitate könnte schier endlos ausfallen, um dies zu untermauern. Dabei ist auffällig,

${ }^{4}$ Zum Übergang von der Rekonstruktion der Gedächtnisorte zu deren Dekonstruktion vgl. Les lieux de mémoire, hrsg. von Pierre Nora, 7 Bde., Paris 1984-1992, hier: III: Les France, Bd. 3, 1992, S. 13 ff.; siehe dazu auch Klaus GrOSSE-KRACHT, Gedächtnis und Geschichte: Maurice Halbwachs - Pierre Nora, in: Geschichte in Wissenschaft und Unterricht 47 (1996), S. $28 \mathrm{f}$.

5 Neben dem siebenbändigen Werk: Les lieux de mémoire (Anm. 4) sei hier vor allem verwiesen auf: Pierre NORA, Zwischen Geschichte und Gedăchtnis, Berlin 1990; weitere Literatur bei GROSSE-KRACHT (Anm. 4). 
dass sich der Argumentationshaushalt über die Zeiten hinweg kaum verändert hat. Beliebig herausgegriffen sei ein Zitat von Hugo von Hofmannsthal:
„Das [...] ist der Geist der Antike, ein so großes Numen, dass kein ein- zelner Tempel, obwohl viele ihm geweiht sind, es fasst. - Es ist unser Denken selber, es ist das, was den europäischen Intellekt geformt hat. [...] Ohne Platon und Aristoteles nicht Augustin noch Thomas. [...] Es ist der Mythos unseres europäischen Denkens, die Kreation unserer geistigen Welt. [...] Es ist kein angehäufter Vorrat, der veraltern kőnnte, sondern eine mit Leben trăchtige Geisteswelt in uns selber“.

Und für Paul Valéry stand fest: „Unbedingt europäisch ist alles, was von drei Quellen - Athen, Rom und Jerusalem - herrührt".

Was hier vor allem seit dem 19. Jahrhundert - im Übrigen durchaus auch im nationalsozialistischen Deutschland zum Teil mit entsprechenden unheilvollen ideologischen Konnotationen ${ }^{6}$ - gedacht und gesagt wurde, findet seine Fortsetzung etwa dort, wo der Rheinische Merkur sein 50-jähriges Bestehen im März 1996 mit einer 36-seitigen Sonderbeilage feierte unter dem Titel: „Wurzeln. Die Grundlagen der europäischen Kultur und Zivilisation in der Welt der Antike". Auf der gleichen Linie argumentierte - um nur noch ein weiteres Beispiel zu zitieren - Konrad Adam in der Frankfurter Allgemeinen Zeitung, um eine Lanze für den altsprachlichen Unterricht an den Gymnasien zu brechen:
„Wenn man im Ernst bei Schülern so etwas wie einen europäischen Pa- triotismus wachrufen möchte, wird man auf diese Fächer [ $=$ Latein und Altgriechisch] nicht ganz und gar verzichten können. Europa ist eine Erfindung der Griechen, aus der die Römer etwas Handfestes gemacht haben, einen Staat nămlich, dessen Grenzen bis heute kulturell stilbildend wirken"s?

Wäre es wirklich so simpel und selbstverständlich, wie es hier behauptet wird, wäre es in der Tat ein leichtes Spiel, über europäische lieux de mémoire im antiken Griechenland $\mathrm{zu}$ sprechen. So eindrücklich und einprägsam das Diktum Konrad Adams aber auch sein mag, so fraglich bleiben gleichwohl seine Grundaussagen. Von der problematischen These der bis heute kulturell stilbildenden Grenzen des Imperium Romanum möchte ich erst gar nicht sprechen, zumal zunächst einmal zu fragen wäre, welche Grenzen Adam da vor Augen hat. Dabei möchte ich keineswegs in Abrede stellen, dass sich darüber diskutieren ließe, inwieweit zwar nicht gerade die räumliche Er-

${ }^{6}$ Eine umfassende ideologiekritische Auseinandersetzung mit der propagandistischen Verwendung des Europagedankens in der Zeit des Nationalsozialismus ist immer noch ein Forschungsdesiderat.

${ }^{7}$ Konrad ADAM, Im Netz. Die gefangene Bildung, in: Frankfurter Allgemeine Zeitung, 20. Dezember 1999, S. 49; vgl. im Übrigen etwa auch Heike SCHMOLL, Latein und Griechisch als Grundlagen der europäischen Identităt, in: Frankfurter Allgemeine Zeitung, 2. Mai 2000, S. 3. 
streckung des Imperium Romanum, aber vielleicht doch die Prinzipien und Mechanismen der römischen Reichsbildung auch einen Vorbildcharakter für ein vereintes Europa haben könnten. Hierüber hat sich erst kürzlich Geza Alföldy in einer Abhandlung allerdings aus einer weitaus differenzierteren Perspektive geäußert ${ }^{8}$. Er legte dabei besonderes Gewicht auf die integrative Kraft von Kultur und Politik im zusammenwachsenden Imperium Romanum und betonte zugleich die allgemeine Prosperität als Resultat der globalisierten Wirtschaft des Römischen Reiches mit einem ausgebauten Verkehrswegenetz, einer einheitlichen Währung und einem Recht auf freie Niederlassung und Arbeitsnahme. Ich möchte diesen Aspekt hier aber nicht weiter vertiefen, zumal man bei solchen Vergleichen allzu rasch Gefahr läuft, sich in vordergründigen Analogien zu verlieren.

Wie aber steht es um die - nicht nur von Konrad Adam aufgestellte - Behauptung, Europa sei eine Erfindung der Griechen? Für die geographische Bezeichnung und Raumvorstellung trifft dies in begrenztem Umfang fraglos zu, auch wenn das Wort selbst wahrscheinlich aus dem Semitischen übernommen wurde. An der syrischen Küste verorteten die Griechen bekanntlich auch die Heimat Europas, der schönen Tochter eines phönikischen Königs, die Zeus in der Gestalt eines Stiers nach Kreta entführte. Ich möchte auf diesen Mythos aber gar nicht näher eingehen, in dem sich die Erinnerung der Griechen an die frühen, sehr engen kulturellen und auch politischen Verbindungen zwischen Griechenland, der Levante und dem übrigen Vorderen Orient widerspiegelt. So besehen war dieser eigentliche und ursprüngliche „europäische“ Mythos zwar für die Griechen der Antike zweifellos so etwas wie ein lieu de mémoire; heute eignet er sich allen Zitaten zum Trotz allerdings kaum, um als lieu de mémoire eines zusammenwachsenden Europa zu dienen, das sich allemal schwer tut, seine südöstlichen Grenzen genauer zu bestimmen.

Auch ist hier nicht der Ort, die Genese des griechischen Europabegriffes nachzuzeichnen, da er mit dem unsrigen kaum mehr als den Namen und eine ungefähre geographische Vorstellung gemeinsam hat ${ }^{9}$. Und diese geographi-

${ }^{8}$ Geza ALFÖLDY, Das Imperium Romanum - ein Vorbild für das vereinte Europa?, Basel 1999; vgl. auch Klaus Martin GIRADET, Bundesstaaten im antiken Griechenland und das römische Imperium als ,supranationale‘ Ordnung - Modelle für ein vereintes Europa von morgen?, in: Europa. Traditionen - Werte - Perspektiven, hrsg. von Roland Martin, St. Ingbert 2000, S. 13-48.

${ }^{9}$ Aus der reichen Fülle einschlägiger Abhandlungen zur Genese des Europabegriffs in der Antike und zur Frage, inwieweit eine der unsrigen vergleichbare Europaidee bereits in der Antike nachzuweisen ist, seien hier nur zwei jüngere, in ihrer Ausdeutung durchaus kontroverse Arbeiten genannt, die auch Hinweise auf weiterfuhrende Literatur enthalten: Justus COBET, Europa und Asien - Griechen und Barbaren - Osten und Westen. Zur Begründung Europas aus der Antike, in: Geschichte in Wissenschaft und Unterricht 47 (1996), S. 405 419; Alexander DEMANDT, Europa: Begriff und Gedanke in der Antike, in: 
sche Vorstellung ist in der Antike bekanntlich auch erst allmählich über Jahrhunderte hinweg gewachsen. Zunächst nur einen kleinen nordöstlichen Teil des griechischen Festlands bezeichnend, wird der Name Europa dann vor allem in der Zeit der Auseinandersetzungen zwischen Griechen und Persern vom 6. bis 4. Jahrhundert v. Chr. auf immer größere Bereiche der nördlichen Mittelmeerwelt ausgedehnt, um schließlich im Weltbild des Claudius Ptolemaios (um $150 \mathrm{n}$. Chr.) von Gibraltar im Westen bis an die Krim und den Ural im Osten und von ,ultima Thule“ im Norden bis nach Kreta und Sizilien im Süden zu reichen.

Diesem geographischen Begriff von Europa korrelierte im antiken Griechenland nie ein entsprechender kultureller oder gar politischer Begriff. Selbst in der Zeit der griechisch-persischen Auseinandersetzungen erwies sich der Europabegriff letztlich als ungeeignet, die zunehmend auch als politischer und kultureller Gegensatz empfundene Konfrontation allein auf die oppositionelle Gegenüberstellung von Asien und Europa zuzuspitzen. Bezeichnend ist eine Anmerkung im Geschichtswerk Herodots: „Asien eignen sich die Perser zu; Europa und die hellenische Welt sind aber nach persischer Auffassung davon abgesondert ${ }^{10}$. Europa und to Hellenikón werden hier differenziert, da beide Begriffe sowohl in geographischer als auch in politischer und kultureller Hinsicht nur zum geringeren Teil als deckungsgleich empfunden wurden. Noch deutlicher wird das bei Aristoteles, der die Griechen in der Mitte zwischen Asien und den kalten Regionen Europas ansiedelt ${ }^{11}$. Ein so weit gefasster und eben vor allem geographischer Begriff taugt daher selbst im weiteren Sinne ebenso wenig als Fokus zur Bestimmung antiker europäischer lieux de mémoire wie der Mythos von der phönikischen Prinzessin aus dem fernen Syrien - es sei denn, man nutzt sie zur Dekonstruktion eines vielleicht dann doch zu engen und starren Europabegriffs.

Mit dieser Einschränkung berühre ich aber die eigentliche Problematik bei der Bearbeitung der Frage nach europäischen lieux de mémoire im antiken Griechenland. Eine angemessene Antwort auf diese Frage setzt zunächst einmal voraus, dass es eine Vorstellung von dem gibt, was in diesem Fall mit „europäisch" gemeint ist. Der Leitfaden, der zur Vorbereitung auf die Konferenz „,Europäische‘ lieux de mémoire?“" diente, weist über die bloße geographische Verortung europäischer lieux de mémoire hinaus auf deren inhaltliche Bezüge zu einer als Ganzheit verstandenen Geschichte Europas. Hier aber liegt die eigentliche Crux. Denn wenn die „Orte der Erinnerung“ die rerum imagines sind, die das kollektive historische Gedächtnis eines Staates oder auch einer anderen Gemeinschaft formen und in denen solche Kommunitäten

Imperium Romanum. Studien zu Geschichte und Rezeption. Festschrift für Karl Christ zum 75. Geburtstag, hrsg. von Peter Kneissl und Volker Losemann, Stuttgart 1998, S. 137-157.

${ }^{10}$ HeRODOT 1.4.4.

$"$ ARISTOTELES, Politik 1320 b 20 ff.; vgl. hierzu auch DEMANDT, Europa (Anm. 9), S. 145 f: 
ihre Identität finden und historisch legitimiert werden, dann setzt die Identifizierung dieser Orte ein Grundwissen über das Selbstverständnis der Gruppen, oder besser gesagt: ein gewisses Vorverständnis über den Zusammenhalt der Gruppen voraus, die sich dieser Orte bedienen. Jedenfalls müssen aber zumindest einige wesentliche Faktoren fixiert sein, die die jeweilige Gruppe konstituieren. Für das von Pierre Nora initiierte Projekt der Deskription französischer lieux de mémoire ergab sich durch den Bezug auf die französische Nation eine relativ klare und bestimmte Vorgabe, ohne dass von vornherein - auch dialektisch bedingte - Wechsel zwischen dem, der sich erinnert, und dem, was erinnert wird, ausgeschlossen werden. Man kann aber zunächst grundsätzlich von bestimmten Fixpunkten innerhalb eines variablen Bezugssystems ausgehen.

Unter einer nationalstaatlichen Perspektive wäre daher auch die Frage nach lieux de mémoire im antiken Griechenland weitaus leichter zu beantworten gewesen als unter dem europäischem Aspekt. Zur Verdeutlichung sei hier nur ein Beispiel angesprochen, ohne es näher auszuführen. Die Griechenbegeisterung des ausgehenden 18. und des 19. Jahrhunderts war zwar eine europaweite, aber damit noch keineswegs eine europäische Bewegung ${ }^{12}$. Jeder Staat suchte und fand seinen eigenen Zugang zu den antiken Stätten und wies diesen einen Platz in seinem kollektiven Gedächtnis zu. Die Entdeckung der griechischen Antike war ein wichtiges Element nationaler Selbstvergewisserung im 19. Jahrhundert; hiervon zeugt exemplarisch die Geschichte der großen nationalen archäologischen Ausgrabungen jener Zeit, die ein Spiegelbild dieses Prozesses abgibt ${ }^{13}$. Die Archäologen schufen neue lieux de mémoire, indem sie diese entdeckten. Sinnsuche und Sinngebung standen dabei in einem untrennbaren dialektischen Bedingungsgefüge. Mochten auch Ausgangs- und Zielpunkte bei allen Nationen im Wesentlichen gleich gewe-

12 Vgl. zum Folgenden mit weiterführender Literatur: Wilhelm BARTH/Max KEHRIGKORN, Die Philhellenenzeit. Von der Mitte des 18. Jahrhunderts bis zur Ermordung Kapodistrias' am 9. Oktober 1831, München 1960; Regine QUACK-EuSTATHIADES, Der deutsche Philhellenismus während des Freiheitskampfes 1821-1827, München 1984; Europäischer Philhellenismus. Ursachen und Wirkungen, hrsg. von Evangelos Konstantinou und Ursula Wiedemann, Neuried 1989; Europäischer Philhellenismus. Die europäische Literatur bis zur 1. Hälfte des 19. Jahrhunderts, hrsg. von Evangelos Konstantinou, Frankfurt a. M. [u. a.] 1992; Der Philhellenismus in der westeuropäischen Literatur 1780-1830, hrsg. von Alfred Noe, Amsterdam/Atlanta 1994; Die Rezeption der Antike und der europäische Philhellenismus, hrsg. von Evangelos Konstantinou, Frankfurt a. M. [u. a.] 1998; George MARGARITIS, Griechenland. Wiedergeburt aus dem Geist der Antike, in: Mythen der Nationen. Ein europäisches Panorama, hrsg. von Monika Flacke, Berlin 1998, S. 152173; Gerhard GRIMM, „We are all Greeks“. Griechenbegeisterung in Europa und Bayern, in: Das neue Hellas. Griechen und Bayern zur Zeit Ludwigs I., hrsg. von Reinhold Baumstark, München 1999, S. 21-32.

${ }_{13} \mathrm{Vgl}$. hierzu etwa die ideologiekritische Untersuchung von Suzanne L. MARCHAND, Down from Olympus. Archaeology and Philhellenism in Germany, 1750-1970, Princeton 1996. 
sen sein, so verharrte man doch stets im Rekurs auf die eigenen nationalen Belange. Zu Beginn des 19. Jahrhunderts erschlossen sich die Eigenarten der griechischen Poliswelt den in den Dimensionen ihres Empires denkenden Briten anders als den Deutschen, die in der Vielgestaltigkeit der griechischen Staatenwelt ein verbindendes Element sahen, das als tertium comparationis den Gedanken einer griechisch-deutschen Verwandtschaft stützen konnte. Beherrschend war (zunächst noch) die Idee von einer durch die gemeinsame Sprache und Kultur verbundenen Nation, deren kleinstaatliche Binnenstruktur eher eine notwendige Voraussetzung als ein störendes Hindernis darstellte gerade so, wie man sich die Gegebenheiten in der griechischen Poliswelt der klassischen Zeit vorstellen zu können glaubte ${ }^{14}$.

Eine europäische Dimension erlangte der Philhellenismus allenfalls in der Zeit der griechischen Freiheitskriege ab 1821, als sich allenthalben in Europa Kräfte regten, um die Griechen in ihrem Kampf gegen die osmanische Herrschaft zu unterstützen. Die europäische Dimension blieb aber letztlich sowohl von ihrem Ursprung her wie auch im Ergebnis nationalstaatlich orientiert. Die lieux de mémoire der antiken griechischen Geschichte von Marathon, den Thermopylen und Salamis über Delphi und Olympia bis nach Chaironeia, die in den westeuropäischen Staaten und auch in den USA zu historischen Orientierungspunkten geworden waren, wurden nun als solche auf Griechenland rückprojiziert und zur schöpferischen Grundlage einer neuen nationalen griechischen Identität gemacht. Es sei nur am Rande bemerkt, dass dies eigentlich ein contradictio in se war, da der Hellenenname in der Antike zu keiner Zeit mit einer nationalstaatlichen Konnotation versehen war ${ }^{15}$. Aus den Bewohnern des Landes, die sich in der Nachfolge des untergegangenen Byzantinischen Reiches stolz als Rhomioi - „Römer“- bezeichneten, wurden nun wieder Hellenen gemacht, was Jacob Philipp Fallmerayer zu der spöttischen und damals vielfach Empörung hervorrufenden Bemerkung veranlasste, dass doch eigentlich kaum noch ein Tropfen althellenischen Blutes in den Adern der Bewohner des Landes fließe ${ }^{16}$. Bis heute ist die (neu)griechische Gedächtnislandschaft von einem tiefen Zwiespalt zwischen einer oft immer noch geradezu zwanghaften Rückbesinnung auf die antiken Wurzeln und

14 Peter FUNKE, Das antike Griechenland - eine gescheiterte Nation? Zur Rezeption und Deutung der griechischen Geschichte in der deutschen Altertumswissenschaft des 19. Jahrhunderts, in: Storia della Storiografia 33 (1998), S. 17-32.

${ }^{15}$ Hierzu immer noch grundlegend Hans Erich STIER, Die geschichtliche Bedeutung des Hellenennamens, Köln/Opladen 1970.

16 Jacob Philipp FALLMERAYER, Welchen Einfluss hatte die Besetzung Griechenlands durch die Slaven auf das Schicksal der Stadt Athen und der Landschaft Attica? Oder nähere Begründung der im ersten Bande der „Geschichte von Morea während des Mittelalters“ aufgestellten Lehre über die Entstehung der heutigen Griechen: gelesen in der öffentlichen Sitzung der kőniglich-bayerischen Akademie der Wissenschaften, Stuttgart/Tübingen 1835 . 
einer fast völligen Ignoranz der eigenen mittelalterlichen und frühneuzeitlichen Geschichte geprägt.

Jedoch geraten wir damit schon wieder viel zu weit in die Gefilde der lieux de mémoire nationaler Prägung. Wie aber lässt sich Noras Ansatz auf eine europäische Fragestellung übertragen? Die Problematik eines solchen Unterfangens hat Nora selbst gesehen. Er hat sich immer dagegen verwahrt, in der Geschichte der französischen Gedächtnisorte nur eine Verteidigung der nationalen Mythen Frankreichs zu sehen. Vielmehr betrachtet er seine Inventarisierung der französischen Gedächtniskultur als eine Materialvorlage auch für einen internationalen Vergleich. Was er dabei im Blick hat, sind mémoires comparées $^{17}$, Elemente einer vergleichenden Geschichte europäischer Gedächtnisorte. Die Themenstellung der Konferenz weist aber darüber hinaus, indem der prinzipiell nationalstaatliche Bezug des Nora'schen Ansatzes auf eine gesamteuropäische Perspektive ausgeweitet wird: Der Blick richtet sich auf europäische lieux de mémoire eben nicht im nationalstaatlichen Vergleich oder als Addition nationaler Gedächtnisorte, sondern als konstitutive Faktoren einer spezifisch europäischen Gedächtniskultur.

Hier beginnen aber die eigentlichen Schwierigkeiten. In dem eben skizzierten Beziehungssystem zwischen dem, der sich erinnert, und dem, was erinnert wird, bleibt der Fixpunkt des Erinnerungssubjekts - eben das „Europäische" - sehr unbestimmt, so dass auch die Frage nach dem Erinnerungsobjekt und seiner materiellen Verortung zwangsläufig vage bleiben muss. Das gilt jedenfalls für eine vorrangig historisch orientierte und historisch argumentierende Erschließung der Fragestellung. Solange die Entscheidung auch politisch noch offen ist, in welchen Grenzen und auf welchen Grundlagen letztlich ein vereintes Europa verwirklicht werden wird, das Kollektiv, das sich erinnern soll, sich noch gar nicht endgültig herausgebildet hat, kann es eigentlich auch noch keine kollektive historische Erinnerungskultur geben - wenigstens nicht als historisch begreifbares Objekt. Zum gegenwärtigen Zeitpunkt bleibt daher die Bestimmung dessen, was europäische lieux de mémoire sein „könnten“ (so ja auch die vorgegebene Themenstellung der Konferenz), von der subjektiven Einschätzung des Betrachters und dessen Europabegriff abhängig. Damit schlüpft der Historiker aber unversehens in eine politische Rolle und wird selbst zum Baumeister und Gestalter einer europäischen Gedächtnislandschaft. Eine solche politische Rolle ist zwar weder neu noch grundsätzlich verwerflich; sie gehört in gewisser Weise sogar zum Beruf des Historikers - zumindest solange sie kritisch positioniert bleibt. Man muss sich aber dieser Rolle bewusst sein, wenn man sich darauf einlässt, das von Pierre Nora entwickelte Deutungsmuster auf eine europäische Dimension zu übertragen.

17 So der Titel eines Themenheftes der von Pierre Nora herausgegebenen Zeitschrift Le Débat 78 (1994); vgl. im Übrigen GROSSE-KRACHT, Gedăchtnis (Anm. 4), S. 29 f. 
Ich möchte mich mit diesen Ausführungen auch keineswegs der eigentlichen Aufgabe entziehen, nach europäischen lieux de mémoire in der griechischen Antike zu fahnden. Ich wollte aber doch meine Schwierigkeiten bei der Bearbeitung der Fragestellung und meine daraus resultierenden Vorbehalte formulieren. Mir schien dies auch um so mehr angebracht, als gerade das antike Griechenland - wie eingangs angemerkt - anscheinend unbestritten und fraglos als fester Bestandteil einer europäischen Geschichts- und Gedächtnislandschaft gilt.

Bei einer genaueren Betrachtung stellt sich der Sachverhalt allerdings doch schwieriger dar. Zumindest sahen das so auch einige Teilnehmer eines Kongresses, der im Oktober 1999 im Gebäude des Europaparlaments in Brüssel tagte, um die Gründung eines „Europa-Museums“ im Jahr 2003 vorzubereiten. Auf der Tagung, an der zahlreiche Wissenschaftler und Politiker teilnahmen - darunter der Kommissionsvorsitzende Prodi sowie die deutschen Altbundeskanzler Schmidt und Kohl -, wurde das Fazit gezogen, dass die Geschichte Europas, wie wir sie heute kennen, eigentlich erst mit der Kaiserkrönung Karls des Großen begonnen habe. Wie es in den - noch unverbindlichen - Empfehlungen zu Inhalt und Umfang der darzustellenden europäischen Geschichte hieß, sei das moderne Europa der sich unter einem Dach vereinigenden Nationalstaaten ,die Tochter des Katholizismus und der Barbaren“, welche das Weströmische Reich unter sich aufgeteilt hatten. Die griechisch-römische Antike wird in diesen Vorschlägen hingegen nur als Vorstufe und eher randständig abgehandelt, da die Griechen sich im Gegensatz zu den übrigen Völkern, den Barbaren, gesehen hätten - also kein Bewusstsein von der Gemeinsamkeit der Kulturen hatten - und die römischen Bürger Vorrechte gegenüber allen anderen Untertanen des Reiches besaßen. Byzanz wird hier sogar als Gegenmodell - ähnlich dem Islam und Asien - gesehen ${ }^{18}$.

Auch wenn kaum damit zu rechnen ist, dass sich diese - auch aus historischer Sicht kaum haltbaren - Vorstellungen unverändert durchsetzen werden, machen sie doch sehr deutlich, wie ideologiebefrachtet das Problem einer historischen Positionierung Europas immer noch ist. Die genannten Kriterien für die Gestaltung eines künftigen „Europa-Museums“ können aber durchaus

${ }^{18}$ Dazu der Artikel von Andreas KRAUSE, Wo beginnt Europa?, in: Athener Zeitung, 5. November 1999, S. 7; bezeichnend war auch die Reaktion der Griechisch-Orthodoxen Kirche auf diese Museumspläne: In einem Appell an „alle Griechen und vor allem die kulturelle Elite des Landes“ rief. der Ständige Heilige Synod in Athen zu „Mobilisierung und Wachsamkeit" auf. Der Westen müsse verstehen, dass „die geistigen Grundlagen Europas die gesunden Elemente der antiken griechisch-römischen Zivilisation, die griechisch-orthodoxe, byzantinische Kultur, die griechisch-christliche oder griechisch-orthodoxe ostkirchliche Tradition und das unter deren unzweifelhaftem Einfluss ausgebildete, lateinisch-römische, westkirchliche Erbe“ seien (zitiert nach Athener Zeitung, 12. November 1999); vgl. auch Nikolaos WENTOURIS, Der diachrone Beitrag Griechenlands zur Bildung des europäischen Bewusstseins, in: Neafon 1 (2001), S. 12-20. 
als eine Art Negativliste dienen, um vor ihrem Hintergrund dennoch mögliche Formen von lieux de mémoire in der griechischen Antike ausfindig zu machen. Dabei wäre es allerdings sinnlos zu fragen, welche Personen oder Orte hier unmittelbar europäisch konnotiert sind. In der Antike wäre kein Grieche je auf den Gedanken gekommen, im heutigen Sinne „europäisch“ zu denken und zu handeln, was auch immer wir darunter verstehen mögen. Europa war für die antiken Griechen - wie schon kurz dargelegt - allenfalls eine geographische Bezugsgröße, niemals aber Bezugspunkt politischen Handelns. Das war allenfalls to hellenikón, die griechische Staatenwelt, in der Regel aber die einzelne Polis, die den Griechen als eigentliche patris - „Vaterland“, Heimatstaat - galt; und Poleis gab es in klassischer Zeit mehr als 800. Alle Versuche, im politischen Handeln der antiken Griechen so etwas wie eine europäische Idee, ein „concetto di Europa“, oder den Wunsch nach einem „grando stato europeo" auszumachen ${ }^{19}$, erweisen sich daher als untauglich ${ }^{20}$.

Dass sich aber dennoch Mythen, Orte und auch Personen der griechischen Antike „europäisieren“ lassen, steht ganz außer Frage. Ich möchte diesen Weg hier aber nicht beschreiten, da er vorab eine entsprechende „europäische" Idee voraussetzt und dann allzu rasch zu einer bloßen Instrumentalisierung zu verkommen droht und Gefahr läuft, sich in völliger Beliebigkeit zu verlieren - gerade weil das Arsenal der griechischen Geschichte so überreich gefüllt ist mit Objekten, die sich für eine (aber eben nicht nur) „europäische“ Aus- bzw. Umdeutung anbieten. Für was alles haben in dieser Hinsicht nicht schon Sparta und Athen, Delphi und Olympia, Miltiades, Themistokles, Leonidas und Perikles - von Homer, Aischylos und Sophokles oder Sokrates, Platon und Aristoteles ganz zu schweigen - herhalten müssen! So besehen, mögen sie in einem Europa-Museum verzichtbar sein, zumal wenn sie als bloße Versatzstücke und Nachwehen eines überkommenen Philhellenismus und Klassizismus daherkommen.

Es sei in diesem Zusammenhang jedoch klar hervorgehoben, dass hiermit nicht in Abrede gestellt wird, dass das, was heute ein (in welchen Grenzen auch immer) vereintes Europa auszeichnet, in vielerlei Hinsicht auf antiken Traditionen beruht und tief im antiken Erbe verwurzelt ist ${ }^{21}$. Ohne die stete

19 Amoldo MOMOGLIANO, L'Europa come concetto politico presso Isocrate e gli Isocratei, in: Rivista di Filologia e d'Istruzione Classica 61 (1933), S. 494; vgl. auch - allerdings vorsichtiger abwägend - COBET, Europa und Asien (Anm. 9).

${ }^{20} \mathrm{Vgl}$. hierzu auch Manfred FUHRMANN, Europas fremdgewordene Fundamente. Aktuelles zu Themen aus der Antike, Zürich 1995, S. 40: „Zwar verwendet schon Herodot, der ,Vater der Geschichte‘, zu Beginn seines Werkes die Namen Europa und Asien, um hiermit zwei grundverschiedene Lebens- und Kulturräume zu bezeichnen. [...] Trotzdem darf man behaupten, dass die Antike ohne einen Europagedanken ausgekommen ist: Die Welt der Griechen und Römer war mediterran, so dass es schon an der geographischen Basis fehlte“.

${ }^{21}$ In diesem Zusammenhang hat zuletzt noch wieder Richard SCHRODER, Einheit der Vielfalt. Europa - was ist das?, in: Frankfurter Allgemeine Zeitung, 14. August 2001, S. 7, hingewiesen auf die Aufzählung von spezifisch Europäischem bei Max WEBER, Vorbemerkung, 
Vergewisserung dieser weit in die Antike zurückreichenden Bindungen verlöre Europa zweifellos ein Stück seiner kulturellen Identität; gleichwohl scheint auch diese Annahme keineswegs mehr unstrittig und selbstverständlich zu $\operatorname{sein}^{22}$. Die nähere Bestimmung dieses griechisch-römischen Erbes Europas steht jedoch auf einem anderen Blatt und ist im Zusammenhang der Fragestellung des Kongresses, soweit diese auf von ihrem Wesen her bereits europäisch konnotierte lieux de mémoire in der griechischen Antike abzielt, nicht zu erörtern.

Streng genommen ist die Frage nach „europäischen“ lieux de mémoire in der griechischen Antike also abschlägig zu beantworten. Anders aber verhält es sich, wenn man nach lieux de mémoire für Europa, also nach konstitutiven Faktoren einer spezifisch europäischen Gedächtniskultur, im antiken Griechenland sucht. Unter diesem Blickwinkel wird dann auch die Frage nach dem griechisch-römischen Erbe wieder relevant, ohne dass allerdings die im Vorangegangenen dargelegten Prämissen außer Betracht bleiben dürfen.

Einfache Antworten sind angesichts der Komplexität einer solchen Betrachtungsweise jedoch auch hier nicht zu finden; und es soll daher im Folgenden nur ein Aspekt exemplarisch herausgegriffen werden, um abschlieBend zumindest die Richtung zu weisen, in welche lieux de mémoire für Europa im antiken Griechenland zu verorten sind. Ich habe hier die originär politischen Dimensionen der griechischen Antike im Blick, die unter europäischer Perspektive eine neue Aktualität gewinnen und im weiteren Sinne durchaus als lieux de mémoire betrachtet werden können - auch im Sinne Pierre Noras, der im Laufe seines Projektes den materiellen Aspekt der Gedächtnisorte immer weiter gefasst hat und schließlich auch kollektive Abstrakta sowie geistige, ökonomische und politische Prozesse in seine Materialsammlung mit aufgenommen hat ${ }^{23}$. Ich möchte das, was ich unter den politischen Dimensionen verstehe, abschließend knapp skizzieren, ohne ins Detail zu gehen. Dabei kommt es mir vor allem auf zwei Aspekte an:

Der erste betrifft das, was Jochen Martin die „Verstaatlichung der Polis“ und Christian Meier die „Entstehung des Politischen“ genannt haben ${ }^{24}$. Vor dem Hintergrund hier nicht näher zu erläuternder, tiefgreifender politischer und sozialer Umbrüche erschlossen sich die Griechen in archaischer Zeit mit der Polis nicht nur einen neuen Lebens-, sondern zugleich auch einen neuen

in: DERS., Gesammelte Aufsätze zur Religionssoziologie, Bd. 1, Tübingen 1920, S. 1 ff.

${ }^{22} \mathrm{Zu}$ dieser Problematik jüngst noch Egon FLAIG, Unsere fremd gewordene Antike. Warum wir ihr mehr verdanken, als wir noch wahrhaben wollen, in: Neue Zürcher Zeitung, 6. Oktober 2001, S. 85; vgl. im Übrigen auch die grundlegenden Überlegungen von Arbogast SCHMITT, Die griechische Antike und das moderne Europa, in: Griechenland in Europa, hrsg. von Gilbert H. Gomig [u. a.], Frankfurt a. M. [u. a.] 2000, S. 9-37.

${ }^{23}$ GROSSE-KRACHT, Gedächtnis (Anm. 4), S. 29 f.

24 Jochen MARTIN, Zur Entstehung der Sophistik, in: Saeculum 27 (1976), bes. S. 154 ff.; Christian MEIER, Die Entstehung des Politischen bei den Griechen, Frankfurt a. M. ${ }^{3} 1995$. 
politischen Raum, der zum einzigen Bezugspunkt politischen Handelns wurde und zum bestimmenden Merkmal der politischen Identität seiner Bewohner, der politai (,Bürger"). Die Ablösung der alten gentilizischen Strukturen ging einher mit der politischen Institutionalisierung der Polis. Rat und Volksversammlung wurden in ein festes Regelwerk eingebunden, dessen schriftliche Fixierung zusätzliche Rechtssicherheit schuf ${ }^{25}$.

Die Formalisierung der politischen Entscheidungsprozesse verband sich eng mit der Frage der politischen Teilhabe. Die Auseinandersetzungen um diese Frage, die in den einzelnen Poleis ganz unterschiedliche Lösungen hervorbrachte, macht deutlich, dass Politik zu einem Objekt geworden war, dessen Verfügbarkeit eine Frage der Macht war, das grundsätzlich aber in der Gestaltungsgewalt der Bürger lag. Die jeweils zeitgenössischen politischen Schlagworte der eunomia (,angemessene Zuteilung“), isonomia (,gleichmäßige Zuteilung“) und demokratia (,Herrschaft des demos = Gesamtbürgerschaft) kennzeichnen in Athen den von Solon über Kleisthenes bis Perikles führenden Weg einer steten Erweiterung des Kreises der Bürger, denen uneingeschränkt die Teilhabe an allen politischen Entscheidungsprozessen zugestanden wur$\mathrm{de}^{26}$. Als europäischer lieu de mémoire ist aber nicht allein die Verfassungsform der Demokratie attischer Prägung anzusehen, sondern, weiter gefasst, das Phänomen der Politisierung des öffentlichen Raums der Polis und damit auch ihrer Bürger. Hier liegt der eigentliche Ursprung nicht nur der demokratischen Idee, sondern überhaupt des verfassungspolitischen Denkens und Handelns, die wiederum die unabdingbaren Voraussetzungen für die bis heute gültigen Leistungen der Griechen in Kunst, Literatur und Philosophie bildeten.

Ich möchte aber noch einen zweiten, gerade für die europäische Dimension bedeutsamen politischen Aspekt in das imaginäre Museum Europas stellen. Die Politisierung der Polis korrelierte mit der Atomisierung der griechischen Staatenwelt. Das unbedingte Beharren der Griechen auf der Freiheit und Autonomie der Einzelstaaten bedingte dauernde Spannungen und Konkurrenzen und eine stete Destabilisierung der zwischenstaatlichen Beziehungen.

Es hat nicht an Versuchen gefehlt, dieses Defizit zu überwinden und das zwischenstaatliche Miteinander in eine umfassendere, polisübergreifende Ordnung einzubinden. Damit wurde das Problem der politischen Teilhabe in den zwischenstaatlichen Bereich verlagert, was die Entwicklung ganz neuer Formen des politischen Zusammenlebens in einer Vielstaatenwelt erforderlich machte - eine Konstellation, die im gegenwärtigen Europa allenthalben prä-

25 Das früheste Beispiel einer solchen regulierten Polisverfassung ist die als sogenannte Große Rhetra überlieferte spartanische Verfassung des 8./7. Jahrhunderts v. Chr. (PLUTARCH, Lykurgos 6.2).

26 Grundlegend hierzu (mit weiterführender Literatur): Jochen BLEICKEN, Die athenische Demokratie, Paderborn [u. a.] ${ }^{4}$ 1995; Mogens Herman HaNSEN, Die Athenische Demokratie im Zeitalter des Demosthenes. Struktur, Prinzipien und Selbstverständnis, Berlin 1995. 
sent ist. Alle Bemühungen, möglichst viele Poleis in eine eher locker gefügte koiné eiréne (,allgemeine Friedensordnung") zu integrieren, erwiesen sich allerdings letztlich als Fehlschlag ${ }^{27}$. Ein weitaus größerer Erfolg war hingegen der Ausbildung wirkkräftiger Bundesstaaten beschieden, die im 3. Jahrhundert v. Chr. schließlich die politische Landkarte ganz Griechenlands prägten, als so gut wie alle ehemals eigenständigen Poleis Mitglieder bundesstaatlich organisierter Verbände geworden waren ${ }^{28}$. Die Anfänge reichen aber schon ins 5. und 4. Jahrhundert v. Chr. zurück. Vor allem an den Randzonen der Poliswelt - in Achaia, Aitolien und Akarnanien und besonders früh in Böotien - entwickelten sich neuartige Staatengebilde, die im Hinblick auf die Organisation des zwischenstaatlichen Miteinanders der Poleis zukunftsweisende Formen aufwiesen. Die Schaffung föderativer Staatsstrukturen bot neue Möglichkeiten, die offenkundigen Schwächen der Vielstaatenwelt Griechenlands zu überwinden, da sie die Eigeninteressen der Poleis und die Erfordernisse polisübergreifender Politik in Einklang zu bringen versprachen. Schon in klassischer Zeit waren Bundesstaaten wie der Arkadische Bund, der Boiotische Bund und der Chalkidische Bund neben den zahllosen eigenständigen Poleis ein bedeutender politischer Faktor.

Die antiken Bundesstaaten waren geprägt von dynamischen Wechselbeziehungen zwischen den einzelnen Poleis und der Zentralgewalt. Aufgrund gemeinsamer Vereinbarungen hatten die Poleis als Gliedstaaten einen Teil ihrer eigenstaatlichen Kompetenzen auf die Bundesebene übertragen und in die Verfügungsgewalt des gesamten Bundes gestellt. Das betraf vor allem Fragen der Außen- und Verteidigungspolitik sowie der Wirtschaft und Finanzen, aber auch große Bereiche der Gesetzgebung und der Rechtsprechung. Die Kompetenzbereiche des Bundes und der Gliedstaaten waren dabei nicht immer scharf voneinander getrennt, sondern konnten durchaus - wie auch in modernen Bundesstaaten - in einem konkurrierenden Verhältnis zueinander stehen und bedurften dann einer wechselseitigen Abstimmung. Wie in den einzelnen Gliedstaaten, gab es auch auf der Bundesebene eigene Magistrate und Entscheidungsorgane (Bundesversammlungen als Primärversammlungen und Bundesräte als Repräsentativorgane). Die Mitwirkung jedes Bürgers auch

${ }^{27}$ Martin JEHNE, Koine Eirne. Untersuchungen zu den Befriedungs- und Stabilisierungsbemühungen in der griechischen Poliswelt des 4. Jahrhunderts v. Chr., Stuttgart 1994.

${ }^{28}$ Zur Geschichte der griechischen Bundesstaaten (mit weiterfuhrender Literatur): Jakob A. O. LARSEN, Greek Federal States. Their Institutions and History, Oxford 1968; Hans BECK, Polis und Koinon. Untersuchungen zur Geschichte und Struktur der griechischen Bundesstaaten im 4. Jahrhundert v. Chr., Stuttgart 1997; Peter FUNKE, Die Bedeutung der griechischen Bundesstaaten in der politischen Theorie und Praxis des 5. und 4. Jh. v. Chr. Auch ein Kommentar zu Aristot. pol. 1261a22-29, in: Theorie und Praxis der Politik im Altertum, hrsg. von Wolfgang Schuller, Darmstadt 1998, S. 59-71; GIRADET, Bundesstaaten (Anm. 8); Gustav Adolf LEHMANN, Ansätze zu einer Theorie des griechischen Bundesstaates bei Aristoteles und Polybios, Göttingen 2001. 
an den Entscheidungen auf der Bundesebene wurde dadurch garantiert, dass er zusammen mit dem Erwerb des Bürgerrechts eines Gliedstaates immer auch das Bundesbürgerrecht erhielt (,doppeltes Bürgerrecht"). Die Bundesversammlungen, an denen in der Regel alle Bürger teilnehmen konnten, verloren angesichts der Größe vieler Bundesstaaten im Laufe der Zeit vielfach gegenüber den Bundesratsgremien an Bedeutung, in denen die Gliedstaaten proportional zu ihrer Größe durch Abgeordnete vertreten waren. So wurde bereits in der griechischen Antike das bundesstaatliche Prinzip mit den Ideen von Proportionalität und Repräsentative verbunden, die heute zu den Grundgedanken des modernen Parlamentarismus gehören und auch von entscheidender Bedeutung für die Schaffung einer politischen Einheit Europas sind.

Nachdem die Römer ihre Herrschaft über die östliche Mittelmeerwelt ausgedehnt hatten, verloren die hier nur überaus knapp skizzierten politischen Entwicklungen in der griechischen Staatenwelt ihre Dynamik und kamen schließlich sogar ganz zum Stillstand, so dass sie in ihrer Zeit längerfristig keine nachhaltigen Wirkungen mehr entfalten konnten. Das mindert allerdings nicht deren Relevanz und Modellcharakter für ein auch politisch zusammenwachsendes Europa. So besehen, stellt die Entwicklung neuer politischer Strukturen im antiken Griechenland wenn schon nicht einen europäischen lieu de mémoire, so doch einen (und keineswegs den einzigen) lieu de mémoire für Europa dar.

\section{Summary}

The search for actual "European" lieux de mémoire in ancient Greece appears to be in vain, since the ancient geographical representation of Europe was never connoted culturally or even politically. In contrast to the national movements of the 19th century, for which ancient Greece was an important element of self-assertion, a European movement in politicis cannot therefore make reference to ancient Greek lieux de mémoire. However, we can look for lieux de mémoire for Europe, i. e. for constitutive factors of a specific European memory culture, in ancient Greece. Yet even so, one does not find lieux de mémoire in the sense of materially tangible places, but at most in the sense of collective abstracta as well as cultural and political processes. This is demonstrated in the last part of this article with two examples from the political realm: the "emergence of the political" (Chr. Meier) and the development of federal forms of organization according to the principles of proportionality and representation. 